\title{
The relation of PAH strength with cold dust in galaxies ${ }^{\star}$
}

\author{
M. Haas ${ }^{1}$, U. Klaas ${ }^{1}$, and S. Bianchi ${ }^{1,2,3}$ \\ 1 Max-Planck-Institut für Astronomie (MPIA), Königstuhl 17, 69117 Heidelberg, Germany \\ 2 Max-Planck-Institut für Astrophysik, Karl-Schwarzschild-Str. 1, Postfach 1317, 85741 Garching, Germany \\ 3 European Southern Observatory, Karl-Schwarzschild-Str. 2, 85748 Garching, Germany \\ Received 17 January 2002 / Accepted 11 February 2002
}

\begin{abstract}
Mid-infrared spectra and submillimetre maps are investigated for five galaxies covering a range of star forming activity. We find a good spatial coincidence between the $850 \mu \mathrm{m}$ continuum emission and the strength of the PAH $7.7 \mu \mathrm{m}$ line. The PAH $7.7 \mu \mathrm{m}$ peak to $850 \mu \mathrm{m}$ flux ratio lies in the range around 2 with a moderate dispersion across the galaxies. Both PAH and cold dust emission correlate also with the emission from very small grains at $14.3 \mu \mathrm{m}$, but regions with starbursts show an excess in the very small grain emission. This suggests that the PAH carriers are preferentially related to the regions dominated by cold dust and molecular clouds, where they are excited mainly by the interstellar radiation field. The lack of increased PAH/submm ratio in starburst knots suggests that starbursts play a minor role for powering the PAH emission in galaxies.
\end{abstract}

Key words. galaxies: NGC 891, NGC 1569, NGC 6946, NGC 7331, Arp 244 - ISM - infrared: galaxies

\section{Introduction}

Polycyclic aromatic hydrocarbonates (PAHs, review in Léger \& Puget 1989) are thought to be favourably related to star forming activity as traced by the $\mathrm{H}_{\alpha}$ emission (Roussel et al. 2001). In this picture, massive young stars provide the UV photons for exciting the PAH carriers which reside at some distance from the $\mathrm{HII}$ regions. The PAH carriers are destroyed (or experience chemical transformations) in strong UV radiation fields like H II regions themselves (M17, Cesarsky D. et al. 1996). Hence, a complication of this picture is that the same mechanism, massive star formation, causes two opposite effects: $\mathrm{PAH}$ emission and PAH destruction.

With increasing star forming activity some kind of saturation or turn-over in the PAH strength would be expected. E.g. above some threshold in the IRAS F60/F100 ratio, as measure for star forming activity, the PAH strength decreases with respect to the emission of very small grains (VSGs) traced by the mid-infrared continuum (e.g. Helou et al. 1991; Dale et al. 2000; Madden 2000; Hunter et al. 2001). However, as pointed out by Dale et al., this inflection might just be due to increased VSG contribution. Thus, the issue of PAH destruction is not yet solved.

The PAH $7.7 \mu \mathrm{m}$ line to $850 \mu \mathrm{m}$ continuum ratio turns out to be similar for both ultraluminous infrared galaxies

Send offprint requests to: M. Haas,

e-mail: haas@mpia.de

* Based on observations with the JCMT and ISO, an ESA project funded by Member States (especially the PI countries France, Germany, the Netherlands and the United Kingdom) and with the participation of ISAS and NASA.
(ULIRGs) and normal starforming galaxies (Haas et al. 2001; Klaas et al. 2001). This suggests that per unit dust mass (as measured by the $850 \mu \mathrm{m}$ continuum flux) the extreme starbursts in ULIRGs do neither destroy many of the PAH carriers nor do they power the bulk of the $\mathrm{PAH}$ emission.

Resolved observations of the rather quiet edge-on galaxy NGC 891 actually show the extensive PAH distribution to be similar to the one of large dust grains and neutral molecular clouds (Mattila et al. 1999). Also in our Galaxy the PAH features are widely distributed (Mattila et al. 1996), and they have been detected even in isolated cirrus clouds and reflection nebulae with low UV photon density (Lemke et al. 1998; Uchida et al. 2000), providing evidence that no extraordinary excitation conditions are required. However, a minimum radiation field is necessary to excite the PAHs; for example in M31 the star forming activity is very low (Xu \& Helou 1996; Schmidtobreick et al. 2000), as well as the $7.7 \mu \mathrm{m} / 11.3 \mu \mathrm{m}$ line ratio, i.e. the PAH 7.7 $\mu \mathrm{m}$ strength (Cesarsky D. et al. 1998; Pagani et al. 1999). Nevertheless, PAH emission is found throughout this UV-quiet galaxy (Moshir et al. 1999).

Hence, a picture is desired which combines (1) the global correlation between PAH strength and star forming activity with (2) the relation of the PAHs with the quiet ISM. The following working hypothesis may be formulated: even in galaxies with strong star forming activity the bulk of the PAH emission does not come from the starburst regions, rather the PAH carriers are ubiquitously distributed in the ISM where they are excited by the interstellar radiation field (ISRF). In star forming galaxies the ISRF is sufficiently strong. In addition, an enhanced star forming activity leads to increased VSG emission, but 
has only moderate effects on the net PAH output. Locally a destruction of the PAHs in the H II regions may partly compensate for a possible enhancement of $\mathrm{PAH}$ emission in the surrounding. Finally, we do not expect the dust responsible for the $850 \mu \mathrm{m}$ emission to be as easily destroyed as the PAH carriers in intense radiation fields. In order to test this hypothesis, here we study the spatial distribution and intensity of the PAH features and the $850 \mu \mathrm{m}$ continuum (tracing the ISM) for five resolved galaxies with moderate to strong star forming activity for which appropriate maps are available from ISO and SCUBA. If the PAH $7.7 \mu \mathrm{m}$ peak flux to $850 \mu \mathrm{m}$ ratio does not change in the vicinity of starbursts, this would favour the hypothesis.

\section{Data}

The galaxies studied are NGC891 (egde-on Sb/c), NGC 1569 (Irr with starbursts), NGC 6946 (face-on Sc with bright nucleus and star forming complexes in the disk), NGC 7331 (Sb with dust ring), and Arp 244 (colliding galaxy pair "The Antennae"). The data sets used here have been published in the quoted references, but we focus on the relation between PAH, VSG and $850 \mu \mathrm{m}$ emission.

Maps at $850 \mu \mathrm{m}$ with a beam size of $15^{\prime \prime} F W H M$ are available for all five galaxies, obtained with SCUBA at the JCMT: NGC 891 (Alton et al. 1998), NGC 1569 (Lisenfeld et al. 2002), NGC 6946 (Bianchi et al. 2000), NGC 7331 (Bianchi et al. 1998), Arp 244 (Haas et al. 2000). The maps of NGC 891, NGC 6946 and NGC 7331 were recalibrated. The flux calibration uncertainty lies at about $25 \%$.

Mid-Infrared spectra with the low resolution spectrometer ISOPHOT-S (Lemke et al. 1996) between 5 and $12 \mu \mathrm{m}$ and the imaging spectrometer ISOCAM-CVF (Cesarsky C. et al. 1996) with circular variable filter between 5 and $16 \mu \mathrm{m}$ are available in the ISO Data Archive (Kessler et al. 2000): NGC 891 (Mattila et al. 1998; Le Coupanec et al. 1999), NGC 1569 (Madden 2000), NGC 6946 (Helou 1999), Arp 244 (Mirabel et al. 1998). These spectra (Fig. 1) exhibit the prominent PAH features at $6.2,7.7$ and $8.6 \mu \mathrm{m}$. They sit atop a continuum (as estimated for several positions and brightness levels of the spectra from an eyeballfitted line between 5.5 and $9.5 \mu \mathrm{m}$ ) which (at $7.7 \mu \mathrm{m}$ ) has a strength of about $10-30 \%$ of the PAH $7.7 \mu \mathrm{m}$ peak flux. Shortward of $10 \mu \mathrm{m}$ the shape of the spectra is strikingly similar for nuclear regions, active regions in the disks, as well as quiet regions. Longward of $10 \mu \mathrm{m}$ the continuum increases in NGC 1569 and Arp 244 for those regions with starbursts. These show also the highest $7.7 \mu \mathrm{m}$ continuum. For NGC 7331 the PAH nature of the MIR emission is evident from narrow band photometry (Figs. 4-6 in Smith 2000).

The MIR spectra sample only parts of the galaxies; a full coverage is available by ISOCAM broad band maps in the $L W 2$ filter $(6.7 \pm 1.5 \mu \mathrm{m})$, with a photometric uncertainty of $20-30 \%$. For those regions with spectral observations available we find a linear correlation between the PAH $7.7 \mu \mathrm{m}$ line line peak flux and the broad band $6.7 \mu \mathrm{m}$ flux representing line and continuum (see Table 1). Thus

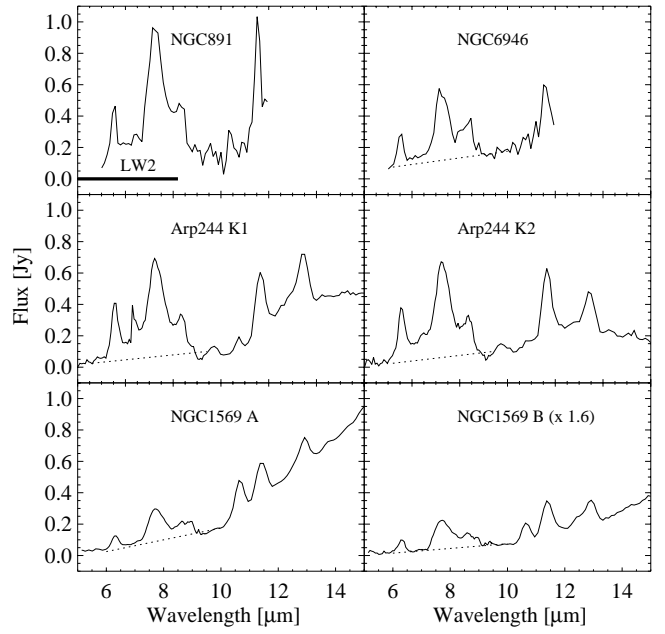

Fig. 1. MIR spectra at dedicated positions of the galaxies, showing the typical PAH features and the slope of the continuum, in particular longwards of $10 \mu \mathrm{m}$. The dotted lines indicate the eyeball-fitted continuum around $7.7 \mu \mathrm{m}$. The horizontal bar indicates the bandpass of the $L W 2$ filter.

Table 1. Average flux ratios for each galaxy.

\begin{tabular}{lrrr}
\hline \hline \multicolumn{1}{c}{ Obj } & $F_{6.7 \mu \mathrm{m}} / F_{7.7 \mu \mathrm{mpeak}}$ & $F_{6.7 \mu \mathrm{m}} / F_{850 \mu \mathrm{m}}$ & $F_{7.7 \mu \mathrm{mpeak}} / F_{850 \mu \mathrm{m}}$ \\
\hline NGC 891 & $0.45 \pm 0.05$ & $0.83 \pm 0.33$ & $1.84 \pm 0.73$ \\
NGC 1569 & $0.60 \pm 0.18$ & $1.08 \pm 0.30$ & $1.80 \pm 0.60$ \\
NGC 6946 & $0.44 \pm 0.08$ & $0.98 \pm 0.35$ & $2.23 \pm 0.80$ \\
NGC 7331 & & $1.07 \pm 0.25$ & $\sim 2.20 \pm 0.80$ \\
Arp 244 & $0.48 \pm 0.07$ & $0.93 \pm 0.32$ & $1.93 \pm 0.70$ \\
\hline
\end{tabular}

the LW2 flux comes essentially from the PAH features, and a variation of the continuum strength plays only a minor role $(<20 \%)$ for this filter.

Highly active starburst regions can be identified on $14.3 \mu \mathrm{m}$ maps. Therefore, we also compare the $\mathrm{PAH}$ and $850 \mu \mathrm{m}$ maps with broad band maps in the ISOCAM LW3 filter at $14.3 \mu \mathrm{m}$ which trace the contribution of VSGs.

\section{Results and discussion}

Figure 2 shows the $850 \mu \mathrm{m}$ maps superimposed with $6.7 \mu \mathrm{m}$ contours (adapted to $15^{\prime \prime}$ resolution). For each galaxy, the morphology at both wavelengths is strikingly similar:

In NGC 891 the $850 \mu \mathrm{m}$ and $6.7 \mu \mathrm{m}$ contours are prominent for the nucleus, two knots, and the extension towards NE. In NGC 6946 the basic flux concentrations are well recognisable above the (noisy) areas with low surface brightness. In NGC 7331 the dust ring outshines the nucleus. The starburst activity of NGC 1569 is inferred from its well known high $\mathrm{H}_{\alpha}$ luminosity. The features resolved at both $850 \mu \mathrm{m}$ and $6.7 \mu \mathrm{m}$ are the bright nucleus (denoted by "A"), a prominent starburst region "B" southeast of "A" and a region " $C$ " between " $\mathrm{A}$ " and " $\mathrm{B}$ ". "C" has a similar MIR spectrum as "B" (therefore it is not shown in Fig. 1). Much of the gas and dust in Arp 244 is concentrated in the overlap region of the two disks. Beside the two nuclei two knots K1 and K2 in the overlap region 

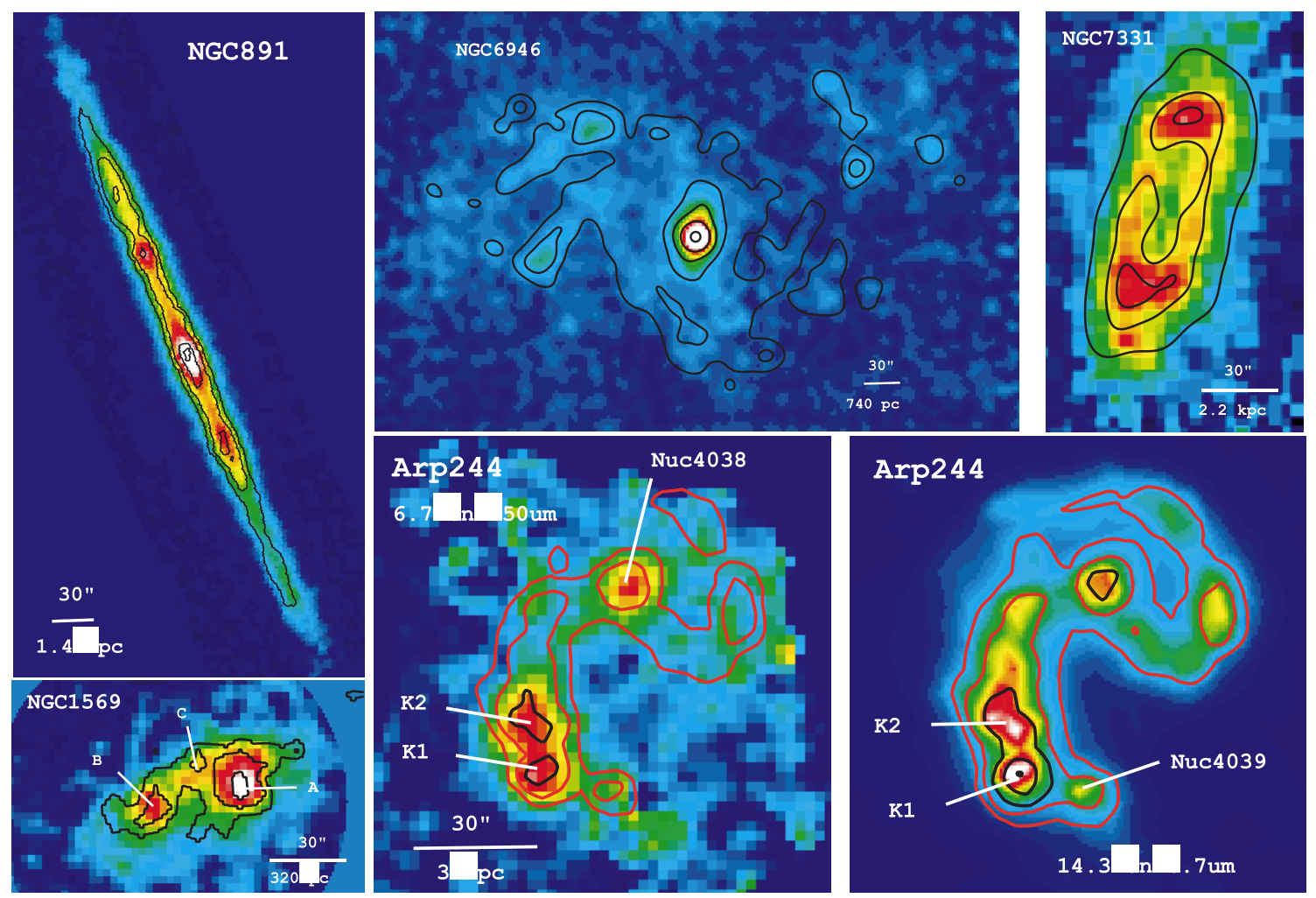

Fig. 2. $850 \mu \mathrm{m}$ maps with $6.7 \mu \mathrm{m}$ contour overlays showing the good spatial coincidence. North is up, east is left. Bottom right for Arp 244: $14.3 \mu \mathrm{m}$ contours on a $6.7 \mu \mathrm{m}$ map, showing the increase of the $14.3 \mu \mathrm{m}$ emission with respect to that at 6.7 and $850 \mu \mathrm{m}$ in the starburst area $\mathrm{K} 1$.

are prominent and of similar brightness in the PAH and submm emission, although they differ in starburst activity (see $14.3 \mu \mathrm{m}$ map).

The morphological coincidence of PAH and submm emission shows up quantitatively via the tight correlation of the fluxes measured in suitably chosen apertures of $15-25^{\prime \prime}$ corresponding to a size of several hundred pc (Fig. 3). For comparison, Fig. 3 also shows the relation with the $14.3 \mu \mathrm{m}$ MIR continuum emission of VSGs.

The PAH $7.7 \mu \mathrm{m}$ line $/ 850 \mu \mathrm{m}$ flux ratios lie in the range around 2 (Table 1 ) and the variations across each of our five galaxies are only moderate, i.e. not of factors of two or more as for the PAH/VSG emission ratio. This range is also comparable to the median ratio of 3.05 found for the nearby ULIRGs (Haas et al. 2001; Klaas et al. 2001). For the five resolved galaxies investigated here the ratio is, with respect to the uncertainties, independent of either a starburst or a more quiet nature.

For NGC 891 the PAH $7.7 \mu \mathrm{m}$ line $/ 850 \mu \mathrm{m}$ flux ratio lies at the low end, which may be explained by a disk viewed edge-on and moderately opaque at $7.7 \mu \mathrm{m}$. Besides extinction some further effects have to be considered: any radio synchrotron contribution at $850 \mu \mathrm{m}$ is clearly below $5 \%$ and can be neglected. Also, the amount of cold dust, the temperature of which may be raised by starbursts, is certainly too small to increase the $850 \mu \mathrm{m}$ flux significantly. Possible CO (3-2) line contribution at $870 \mu \mathrm{m}$ could occur for high excitation conditions in starbursts.
Its contribution to the dust continuum reaches $10 \%$ in NGC 1569 (Lisenfeld et al. 2002) and 30\% in Arp 244 (estimated by Haas et al. 2000). In addition, this effect is partly cancelled out in Fig. 3, since active regions do also show an increased MIR continuum in the LW2 filter (Fig. 1). If the CO (3-2) line contribution would be as high as $50 \%$ in K1 (the most active starburst area of Arp 244), then the PAH emission would be increased with respect to the cold dust mass, but in any case a lot of PAH emission in Arp 244 comes from more quiet regions located far away from $\mathrm{K} 1$.

Both PAH and cold dust emission correlate also (to some extent) with that from very small grains at $14.3 \mu \mathrm{m}$. NGC 891 and NGC 7331 do not house any prominent starbursts and the correlations look well confined in Fig. 3. But those galaxies with starbursts show an excess in the very small grain emission by factors of $2-7$. Such regions are, for example, the nucleus of NGC 6946, the starburst area K1 in the overlapping disks of Arp 244, or a large part of the active dwarf galaxy NGC 1569. Since in these starburst areas the PAH emission is not adequately changed with respect to the $850 \mu \mathrm{m}$ flux, this suggests that the PAH carriers are preferentially related to the regions dominated by cold dust and molecular clouds. There they would be excited mainly by the interstellar radiation field, which in star forming galaxies is sufficiently strong.

Our results refer to a spatial resolution of about several hundred pc in the galaxies studied, thus they average over 


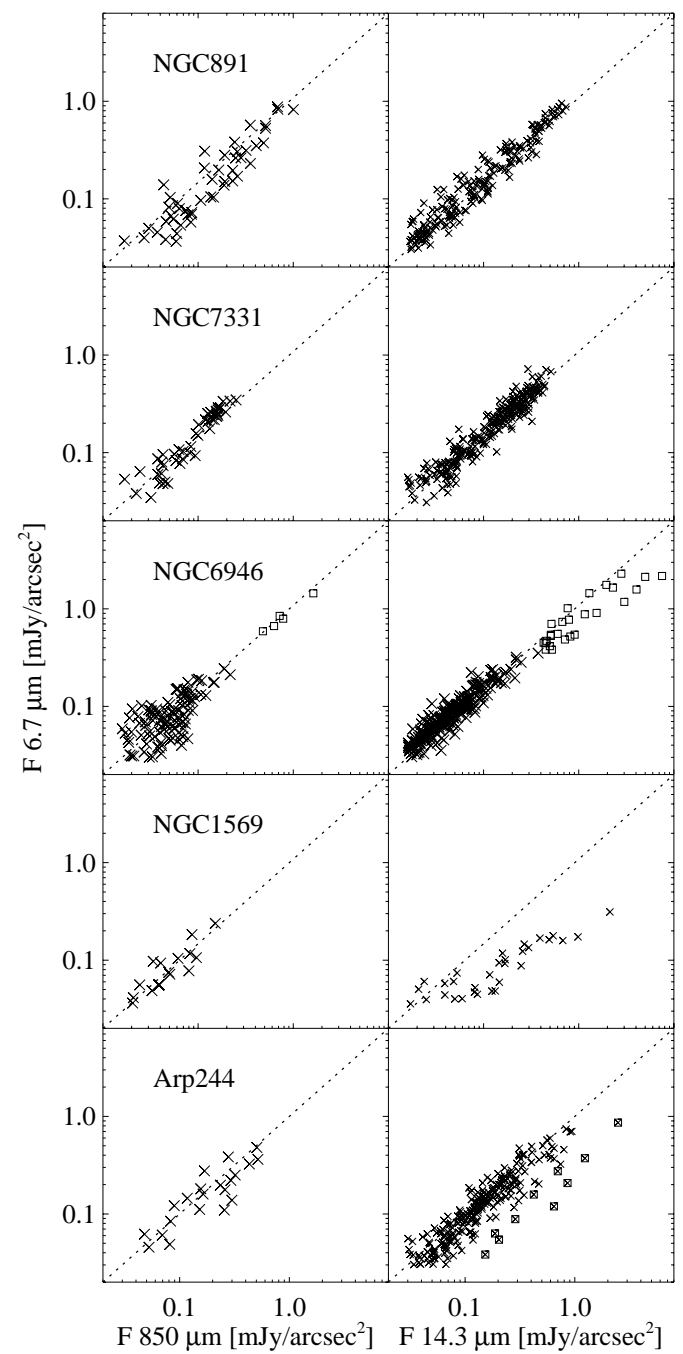

Fig. 3. Relation between the fluxes at $6.7 \mu \mathrm{m}$ versus $850 \mu \mathrm{m}$ (left column) and $14.3 \mu \mathrm{m}$ (right column). Each symbol corresponds to an area in a grid covering the galaxies. The area size is $15-25^{\prime \prime}$ and $6-10^{\prime \prime}$ for the left and right column, respectively. Only those areas with fluxes above the noise threshold $\left(\approx 0.025 \mathrm{mJy} / \operatorname{arcsec}^{2}\right)$ are plotted. The ratio between the fluxes is by chance mostly about unity (indicated by the dotted lines). An exception are the $14.3 \mu \mathrm{m}$ points for the starbursts regions. The nucleus of NGC 6946 and the active knot K1 in the overlap region of Arp 244 are denoted by different symbols.

regions of different activity. Nevertheless, they do not provide any evidence for strong PAH enhancement or destruction in those regions crowded by starbursts. But strong activity clearly increases the VSG emission in the MIR by factors of more than two. In conclusion, the global relation between PAH strength and $\mathrm{H}_{\alpha}$ or other star forming tracers found by various researchers might be only indirect in the sense that those galaxies with a lot of molecular clouds (and PAH emission) more likely form more stars and thus shine brighter in $\mathrm{H}_{\alpha}$. Finally, the finding, that the PAH emission is not mainly powered by starbursts, has an impact on the interpretation of cosmological number counts at $15 \mu \mathrm{m}$, a window the $\mathrm{PAH}$ features move into at $z \approx 0.5$ (e.g. Fig. 1 in Elbaz et al. 1999): the excess of faint sources may not only be due to luminous mergers and starburst galaxies, rather due to more normal (dust and PAH rich) spiral galaxies.

Acknowledgements. We thank the referee Dr. Daniel Dale for his constructive comments. The ISOPHOT Data Centre at MPIA is supported by Deutsches Zentrum für Luft- und Raumfahrt e.V. (DLR) with funds of Bundesministerium für Bildung und Forschung. The authors are responsible for the contents of this publication.

\section{References}

Alton, P. B., Bianchi, S., Rand, R. J., et al. 1998, ApJ, 507, L125

Bianchi, S., Alton, P. B., Davies, J. I., et al. 1998, MNRAS, 298, L49

Bianchi, S., Davies, J. I., Alton, P. B., et al. 2000, A\&A, 353, L13

Cesarsky, C., Abergel, A., Agnese, P., et al. 1996, A\&A, 315, L32

Cesarsky, D., Lequeux, J., Abergel, A., et al. 1996, A\&A, 315, L309

Cesarsky, D., Lequeux, J., Pagani, L., et al. 1998, A\&A, 337, L35

Dale, D. A., Helou, G., Silbermann, N. A., et al. 1999, AJ, 118, 2055

Elbaz, D., Cesarsky, C., Fadda, D., et al. 1999, A\&A, 351, L37

Haas, M., Klaas, U., Coulson, I., et al. 2000, A\&A, 356, L83

Haas, M., Klaas, U., Müller, S. A. H., et al. 2001, A\&A, 367, L9

Helou, G., Ryter, C., \& Soifer, B. T. 1991, ApJ, 376, 505

Helou, G. 1999, ESA-SP, 427, 797

Hunter, D. A., Kaufman, M., Hollenbach, D. J., et al. 2001, ApJ, 553, 121

Kessler, M. F., Müller, T. G., Arviset, C., García-Lario, P., \& Prusti, T. 2000, The ISO Handbook, SAI/2000-035/Dc (ESA publ.)

Klaas, U., Haas, M., Müller, S. A. H., et al. 2001, A\&A, 379, 823

Léger, A., \& Puget, J.-L. 1989, ARA\&A, 27, 161

Le Coupanec, P., Rouan, D., Gallais, P., et al. 1999, ESA-SP, 427, 917

Lemke, D., Klaas, U., Abolins, J., et al. 1996, A\&A, 315, L64

Lemke, D., Mattila, K., Lehtinen, K., et al. 1998, A\&A, 331,742

Lisenfeld, U., Israel, F. P., Stil, J. M., \& Sievers, A. 2002, A\&A, in press

Madden, S. 2000, New Astron. Rev., 44, 249

Mattila, K., Lehtinen, K., \& Lemke, D. 1999, A\&A, 342, 643

Mattila, K., Lemke, D., Haikala, L. K., et al. 1996, A\&A, 315, L353

Mirabel, I. F., Vigroux, L., Charmandaris, V., et al. 1998, A\&A, 333, L1

Moshir, M., Marsh, K., Price, S., \& Shipman, R. 1999, ASP, 177,212

Pagani, L., Lequeux, J., Cesarsky, D., et al. 1999, A\&A, 351, 447

Roussel, H., Sauvage, M., Vigroux, L., \& Bosma, A. 2001, A\&A, 372, 427

Schmidtobreick, L., Haas, M., \& Lemke, D. 2000, A\&A, 363, 917

Smith, B. J. 2000, ApJ, 500, 181

Uchida, K. I., Sellgren, K., Werner, M. W., et al. 2000, ApJ, 530,817

Xu, C., \& Helou, G. 1996, ApJ, 456, 152 